\title{
Effect of Structural and Morphological Changes on the Conductivity of Stretched PANI-DBSA/HIPS Film
}

\author{
Jonghyeok Lee and Eunok Kim* \\ Department of Chemistry, The University of Suwon, Hwaseong-si, Gyeonggi-do 445-743, Korea. ${ }^{*}$ E-mail: eokim@suwon.ac.kr \\ Received April 4, 2011, Accepted June 27, 2011
}

\begin{abstract}
We studied the effect of structural and morphological changes on the conductivity of a stretched conducting polymer film. To improve the poor processability of polyaniline, we used dodecylbenzenesulfonic acid as both a surfactant and a dopant during emulsion polymerization, followed by blending with high-impact polystyrene. UV-Vis/NIR spectra were obtained to observe conformational changes, and SEM and AFM were used to investigate morphological changes. FT-IR dichroism was applied to determine the microscopic orientation, and XRD patterns were obtained for quantitative crystallinity analysis. The electrical conductivity $\left(\sigma \| / \sigma_{\perp}\right)$ was measured as a function of draw ratio. We found a clear correlation between morphological changes and $\sigma_{\|} / \sigma_{\perp}$, especially at the stretching limit. The conductivity of the films can be modified according to the desired application by controlling their structure and morphology.
\end{abstract}

Key Words : Polyaniline, PANI-DBSA/HIPS, Stretch orientation, Morphology

\section{Introduction}

Conducting polymers have the electrical, magnetic, and optical properties of metals, along with their plastic properties. ${ }^{1-4}$ Among the conducting polymers, polyaniline (PANI) has received considerable attention as a promising material, especially for electrical device applications, because of its tunable conductivity and simple doping/dedoping and redox properties. ${ }^{5}$ PANI in the salt form (PANI-ES) is a conductor and is difficult to process because it is insoluble in many common organic solvents and intractable, ${ }^{6}$ whereas PANI in the base form (PANI-EB) is nonconductive but can be made conductive by protonic acid doping.

Various efforts to improve the processability and mechanical properties of PANI-ES have been demonstrated. It is interesting that its poor processability can be improved by polymerization of aniline in the presence of a functionalized protonic acid such as camphorsulfonic acid (CSA) or dodecylbenzenesulfonic acid (DBSA). ${ }^{7}$ In addition, the processability and mechanical properties of PANI-DBSA can be enhanced by blending with commodity polymers. Unlike conventional filler materials, these conducting blends have mechanical properties close to those of insulating commodity polymers. ${ }^{8-12}$

Controlling the conductivity of conducting polymers is very important and is one of the greatest challenges in terms of their application. Both microscopic and macroscopic factors may affect conductivity, and these factors may vary according to the synthetic and processing conditions. ${ }^{13-16}$ Various attempts at conductivity control in conducting films have been performed, for example, doping level control, ${ }^{17,18}$ thickness control, ${ }^{19,20}$ the use of additives such as carbon black or carbon nanofibers, ${ }^{21,22}$ and secondary doping. ${ }^{14,15,23,24}$ Among them, mechanical stretching of the conducting film is one of the most interesting processes for conductivity control. Because PANI-ES is intractable, most of the stretch orientation studies of PANI films have been performed on the more processable PANI-EB, which is subsequently doped with protonic acids. ${ }^{25-28}$ However, Abell et al. opened new possibilities for preparing stretchable PANI-ES film. PANI-ES films were prepared by doping PANI-EB with CSA, yielding PANI-CSA, and cast from solution. ${ }^{29}$

In this work, cast films were prepared by blending PANIES (PANI-DBSA) with commodity polymers, such as styrene polymers, to observe how the conductivity is affected by uniaxial stretching. A PANI-DBSA solution was prepared by emulsion polymerization of aniline. DBSA was used as both a dopant and a surfactant. In PANI-DBSA, the long alkyl chains of the dodecylbenzene group of DBSA give rise to solubility in nonpolar or weakly polar organic solvents, such as toluene, xylene, and chloroform, so that PANI in its conducting form is compatible with commodity polymers having similar molecular structure, such as styrene polymers. ${ }^{7,30}$ Among styrene polymers, high-impact polystyrene (HIPS) can resolve the brittleness problems of polystyrene (PS) by copolymerizing styrene monomers with rubbery butadiene monomers. Therefore, HIPS exhibits good processability and higher elongation at break than PS.

We studied the effect of structural and morphological changes on the conductivity of the stretched conducting polymer films. We found an interrelationship between polymer chain conformation, orientation, crystallinity, morphology, and conductivity. To our knowledge, this is the first study that compares the morphology and the electrical conductivity $\left(\sigma \| / \sigma_{\perp}\right)$ of PANI-DBSA/polystyrene polymer blend films at its stretching limit. In addition, the conductivity level of the films can be modified according to the desired application by controlling the microscopic and macroscopic factors. 


\section{Experimental}

Materials. Aniline monomer (Duksan Pure Chemical, $99 \%$ ) was purified by distillation before use. DBSA, $N$ methylpyrrolidone (NMP), ammonium persulfate (APS), toluene, and ethylene glycol were used as received from Duksan Pure Chemical, without further purification. HIPS resin (Korea Kumho Petrochemical HI425E) was used as received.

Preparation and zone-drawing of PANI-DBSA/HIPS film. In a typical emulsion polymerization, $0.1 \mathrm{~mol}$ aniline and 0.2 mol DBSA were combined with a mixture of 400 $\mathrm{mL}$ of distilled water and $200 \mathrm{~mL}$ of toluene in a $1 \mathrm{~L}$ flask. The mixed solution was cooled in an ice bath and stirred for $30 \mathrm{~min}$. To this solution, an aqueous solution of oxidant 0.08 mol APS in $50 \mathrm{~mL}$ of distilled water was added dropwise with stirring for $15 \mathrm{~min}$. The entire polymerization reaction was conducted at $0{ }^{\circ} \mathrm{C}$ for $15 \mathrm{hr}$. During emulsion polymerization, the color of the solution changed to dark green. After polymerization, the aqueous phase with PANI-DBSA separated into PANI-DBSA in the toluene phase and a colorless aqueous phase. The product cannot be isolated directly, since PANI-DBSA exists in the emulsion along with other by-products. Ethylene glycol was then added to the organic solution of PANI-DBSA in toluene to break the emulsion, and PANI-DBSA was obtained by separation of the supernatant. ${ }^{31,32}$ PANI-DBSA/HIPS solution was prepared by mixing HIPS into a $45 \mathrm{wt} \%$ solution of PANIDBSA in toluene.

PANI-DBSA/HIPS films were cast from the solution on a glass substrate using approximately $5 \mathrm{wt} \%$ of NMP as a plasticizer. ${ }^{33}$ The films (50 mm long, $15 \mathrm{~mm}$ wide, and 0.5 $\mathrm{mm}$ thick; free-standing) were peeled from the glass substrates and stretched up to a draw ratio of $L / L_{0}=9$ by the zone-drawing method $\left(L_{0}, L\right.$ : length before and after stretching, respectively, $L / L_{0}$ : draw ratio) at $180{ }^{\circ} \mathrm{C}$. ${ }^{34}$

Instrumental Analysis. Diffuse reflectance ultravioletvisible/near-infrared (UV-Vis/NIR) spectra were obtained using a Shimadzu UV-3101PC spectrometer. Scanning electron microscopy (SEM) images were taken with a Tescan Korea VEGA 3 LMU instrument operated at $15 \mathrm{kV}$. The SEM samples were gold-sputtered before observation. Atomic force microscopy (AFM) images were made with a Shimadzu SPM-9500J3 instrument in non-contact mode. Fourier trans-

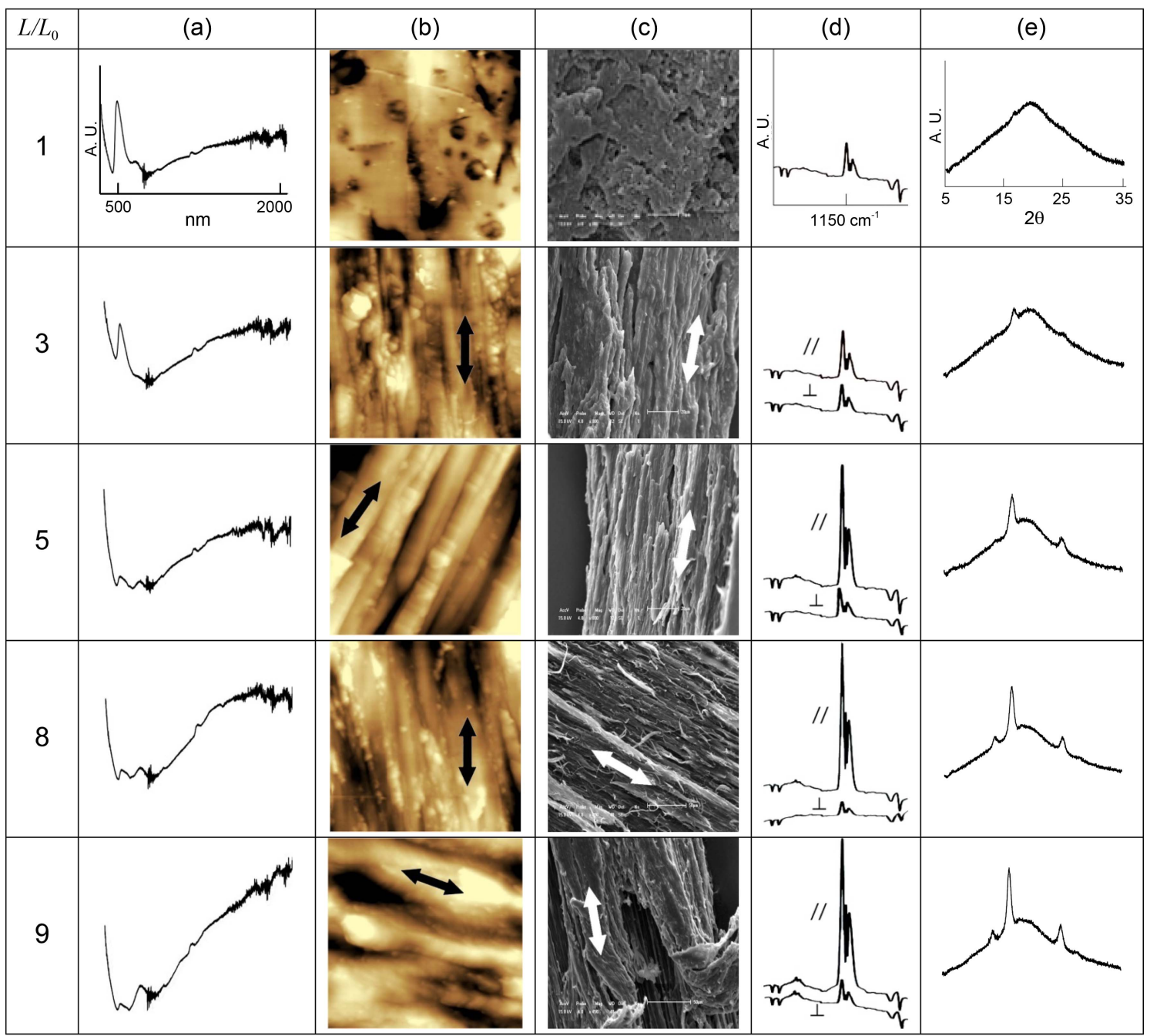

Figure 1. Experimental data on PANI-DBSA/HIPS films with different draw ratios. (a) UV-Vis/NIR spectra, (b) AFM images, (c) SEM images, (d) FT-IR band at $1150 \mathrm{~cm}^{-1}$, (e) XRD patterns. 
form infrared (FT-IR) spectra were registered on a Jasco FTIR 4100 spectrometer with a JASCO ATR PRO470-H attenuated total reflection (ATR) single reflection accessory. $\mathrm{X}$-ray diffraction (XRD) patterns were recorded using an XRD TW3040 X-pert MPD instrument with Ni-filtered Cu radiation $\left(\lambda=1.5418 \AA\right.$ ) by $0.05 \%$ sec scanning at $5^{\circ} \leq 2 \theta \leq$ $35^{\circ}$. The DC conductivity was measured at room temperature by the four-probe method using a Keithley 237 source measurement unit.

\section{Results and Discussion}

The total conductivity of a conducting polymer film depends on both the ability to transport charge carriers along the polymer backbone and the ability of carriers to hop between polymer chains through interpolymer conduction. $^{35,36}$ That is, the conductivity depends on intrinsic factors such as the polymer chain conformation, orientation, morphology, crystallinity, and molecular weight, and extrinsic factors such as the dopants used. ${ }^{5,16,33}$

Figure 1 displays the experimental data (UV-Vis/NIR, AFM, SEM, FT-IR/ATR, XRD) for the films stretched at various draw ratios to the limit of $L / L_{0}=9$. Figure 2 shows the relationship between the conductivity $\left(\sigma_{\|} / \sigma_{\perp}\right)$ and the draw ratio of the film.

Diffuse reflectance UV-Vis/NIR spectroscopy is used to study the polymer chain conformation when determining the conductivity (Figure 1(a)). ${ }^{14,37}$ The unstretched film shows a localized polaron peak at $800 \mathrm{~nm}$; however, this peak decreased and a free carrier tail beginning at $\sim 1000 \mathrm{~nm}$ increased gradually with stretching. The free carrier tail is characteristic of a delocalized polaron band in conductive materials. ${ }^{37}$ The intensity difference of the free carrier tail can be attributed to a difference in the polymer chain conformation and indicates that the conformation changes from a compact coil to an expanded coil. Consequently, the intrachain conductivity increases with increasing $L / L_{0}{ }^{5,37}$ The free carrier tail grows beyond the point of film breakage.

Figure 1(b) and 1(c) show AFM and SEM images, respectively. Double arrows indicate the stretching direction. In the

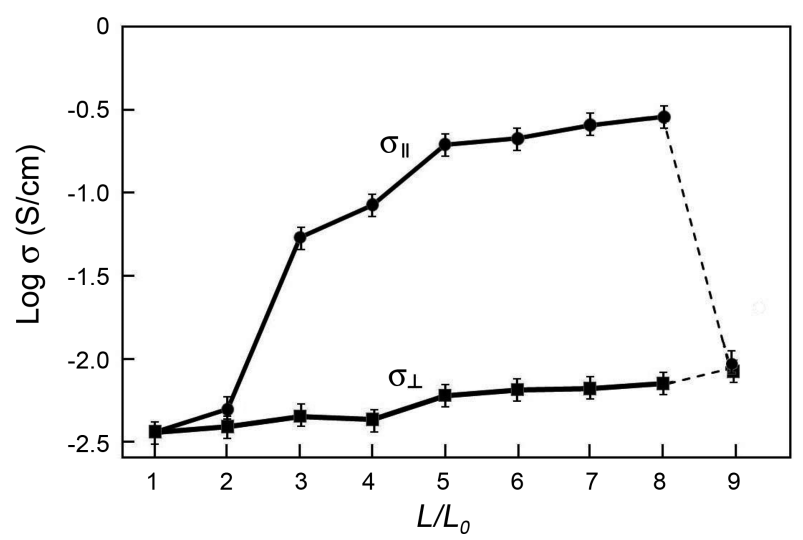

Figure 2. Mean ( \pm 1 standard error) conductivity measurements at three locations. Relationship between conductivity $\left(\sigma_{\|} / \sigma_{\perp}\right)$ and draw ratio. images at $L / L_{0}=3$, two different regions can be distinguished: oriented fibrous structures and the remnants of entangled chains. This indicates that a morphological change from 2-D amorphous to 1-D fibrous structure has begun. With a further increase in draw ratio $\left(L / L_{0}=5\right)$, the film exhibits an increased tendency to fibrillation and appear considerably more regular and highly oriented. At the stretching limit $\left(L / L_{0}=9\right)$, the film slips, and noticeable unidirectional breakage begins to appear. Broken parts appear as dark regions in the AFM image and show fibrous structure in the interface between polymer chains in the SEM image. At this moment, the conductivity in the direction parallel to the stretching direction $(\sigma \|)$ decreases by about two orders of magnitude (Figure 2), which is associated with partial loss of connectivity along the conduction pathways and the film breakage observed by AFM and SEM. ${ }^{14}$ To our knowledge, this is the first observation showing a clear correlation between morphological changes and conductivity in a conducting polymer film at its stretching limit.

We measured changes in polymer chain orientation by FTIR spectroscopy (Figure 1(d)). In principle, certain infrared bands can be used to determine the overall orientation. A strong characteristic band at $\sim 1150 \mathrm{~cm}^{-1}$ is associated with electrical conductivity and acts as a measure of the degree of electron delocalization in PANI. ${ }^{38,39}$ Figure 3 shows changes in the dichroic ratio of the FT-IR band at $\sim 1150 \mathrm{~cm}^{-1}$ as a function of the film's draw ratio. As the polymer chains became uniaxially oriented, the band intensity parallel to the stretching direction $\left(I_{\|}\right)$increased, but that perpendicular to the stretching direction $\left(I_{\perp}\right)$ did not. The dichroic ratio, $R=$ $I_{\|} / I_{\perp}$, may be considered a measure of the directional orientation of the polymer chains. ${ }^{40-42}$ The distinct increase in $R$ confirms that the chains became oriented along the stretching direction with increasing $L / L_{0}{ }^{43}$

The effect of the draw ratio on the development of crystallinity was investigated by XRD (Figure 1(e)). The films were initially strongly disordered, as indicated by the XRD patterns at $L / L_{0}=1$. Three prominent peaks at $2 \theta$ of $\sim 13^{\circ}$, $\sim 17^{\circ}$, and $\sim 25^{\circ}$ began to appear at $L / L_{0}=3$. The intensities of these peaks increased with increasing $L / L_{0}$. The crystallinity was maintained even after breakage of the film occurred.

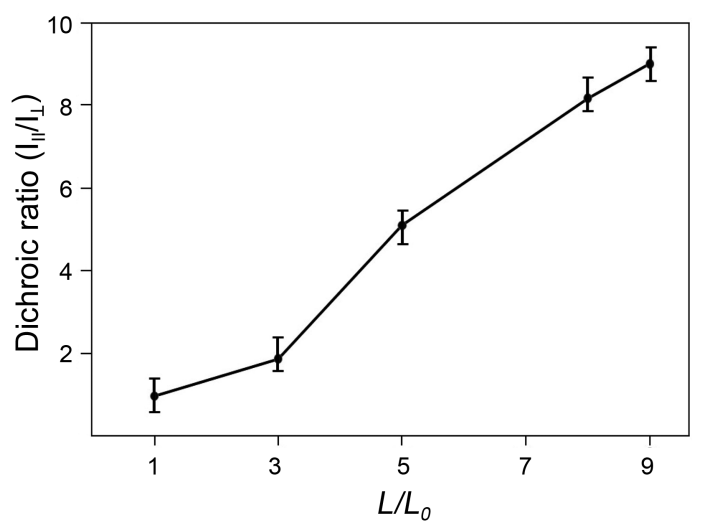

Figure 3. Changes in dichroic ratio of the FT-IR band at $\sim 1150 \mathrm{~cm}^{-1}$. 
Table 1. Relationship between degree of crystallinity and draw ratio

\begin{tabular}{cccccc}
\hline$L / L_{0}$ & 1 & 3 & 5 & 8 & 9 \\
\hline$X_{c}(\%)$ & 0.1 & 4.7 & 9.6 & 12.6 & 12.7 \\
\hline
\end{tabular}

The degree of crystallinity, presented in Table 1, is quantitatively estimated using the method of Nara \& Komiya, ${ }^{44}$

$$
X_{c}=A_{c} /\left(A_{c}+A_{a}\right)
$$

where $X_{c}$ is the degree of crystallinity, $A_{c}$ is the crystallized area, and $A_{a}$ is the amorphous area on the X-ray diffractogram.

A draw ratio of $L / L_{0}=1$ corresponds to $0.1 \%$ crystallinity, whereas $L / L_{0}=9$ produces a crystallinity of $12.7 \%$. As shown in Figure 1(e) and Table 1, crystallization began immediately when these initially amorphous films were stretched. The UV-Vis/NIR, FT-IR, XRD, and conductivity results together indicate that the increased crystallinity is due to the change from the compact coil conformation to the expanded coil conformation, and, in turn, the greater orientation and increased regularity with alignment along the stretching direction. The higher crystallinity resulted in higher inter-chain conductivity, since the inter-chain distance was shortest just before breakage of the film, which indicates that electrons can move easily from one chain to the nearestneighbor chains. ${ }^{37,45}$

To investigate the correlation between the conductivity anisotropy and the draw ratio, we measured the conductivity of the films, both $\sigma \|$ and $\sigma_{\perp}$ (Figure 2). We found that $\sigma \|$ increased with draw ratio up to $L / L_{0}=8$. With further increases up to a maximum draw ratio of $L / L_{0}=9, \sigma \|$ decreased significantly. This result agrees well with the morphological changes observed by AFM and SEM. The conductivity of the unstretched film was $3.64 \times 10^{-3} \mathrm{~S} / \mathrm{cm}$. The conductivity increased dramatically up to $\sigma \| \approx 2.86 \times$ $10^{-1} \mathrm{~S} / \mathrm{cm}$, whereas $\sigma_{\perp} \approx 7.09 \times 10^{-3} \mathrm{~S} / \mathrm{cm}$ at $L / L_{0}=8$. The maximum anisotropy $\left(\sigma_{\|} / \sigma_{\perp}\right)$ was about 40 just before the breakage of the film occurred. This would imply that the films were considerably stretch-oriented. ${ }^{28}$ We propose that the increase in $\sigma \|$ is due to the improvement in intra-chain charge carrier mobility along the PANI backbone. However, $\sigma_{\perp}$ remained almost the same as in the initial state, even though the inter-chain distance closed, since the charge carrier mobility in the parallel direction in the polymer chains plays a dominant role in the conductivity increase. ${ }^{25}$ The results of this work are in good agreement with previous reports regarding the changes in microscopic and macroscopic factors that affect conductivity as a function of draw ratio in PANI film. ${ }^{14,15,27,28}$

\section{Conclusion}

The maximum draw ratio of a PANI film was significantly improved by using DBSA followed by blending with HIPS; draw ratios of up to 9 were achieved in the resulting PANIDBSA/HIPS blend films. Our examination of the films demonstrated the effects of structure and morphology on their conductivity. We also observed an interrelationship between polymer chain conformation, degree of orientation and crystallinity, morphology, and electrical conductivity. In addition, comparisons between the morphology and the conductivity of the conducting polymer-blend films revealed that the loss of conducting pathways dramatically decreased $\sigma \|$ at the limiting draw ratio $\left(L / L_{0}=9\right)$. This work can facilitate conductivity control for a given application by controlling the structure and morphology of a conducting polymer blend.

Acknowledgments. We thank Prof. Eung Ju Oh and Prof. Kwang Sun Ryu for their help with and interest in this work.

\section{References}

1. Skotheim, T. A. Handbook of Conducting Polymers; Marcel Dekker: New York, 1986.

2. Ray, S.; Easteal, A. J.; Cooney, R. P.; Edmonds, N. R. Mat. Chem. Phys. 2009, 113, 829 .

3. Singh, S. K.; Gupta, R. K.; Singh, R. A. Synth. Met. 2009, 159, 2478.

4. Liu, C. C.; Lu, B. Y.; Yan, J.; Xu, J. K.; Yue, R. R.; Zhu, Z. J.; Zhou, S. Y.; Hu, X. J.; Zhang, Z.; Chen, P. Synth. Met. 2009, 160, 2481.

5. Zhang, D. H. Polym. Test. 2007, 26, 9.

6. Ansari, R.; Raofie, F. E-J. Chem. 2006, 3, 35.

7. Cao, Y.; Smith, P.; Heeger, A. J. Synth. Met. 1992, 48, 91.

8. Pud, A.; Ogurtsov, N.; Korzhenko, A.; Shapoval, G. Prog. Polym. Sci. 2003, 28, 1701.

9. Afzal, A. B.; Akhtar, M. J.; Ahmad, M. J. Electron Microsc. 2010 , $59,339$.

10. Ebrahim, S. M.; Soliman, M. M.; Latif, M. M. A. E. High Perform. Polym. 2009, 22, 377.

11. Souza, S. D. Surf. Coat. Technol. 2007, 201, 7574.

12. Pan, W.; He, X.; Chen, Y. Appl. Mech. Mater. 2011, 44, 2195.

13. Jeong, S. K.; Suh, J. S.; Oh, E. J.; Park, Y. W.; Kim, C. Y.; MacDiarmid, A. G. Synth. Met. 1995, 69, 171.

14. MacDiarmid, A. G.; Epstein, A. J. Synth. Met. 1994, 65, 103.

15. MacDiarmid, A. G.; Epstein, A. J. Synth. Met. 1995, 69, 85.

16. Feng, J.; MacDiarmid, A. G.; Epstein, A. J. Synth. Met. 1997, 84, 131.

17. Liu, C.; Zhang, J.; Shi, G.; Chen, F. J. Appl. Polym. Sci. 2004, 92, 171.

18. Kang, Y. K.; Kim, S. K.; Lee, C. J. Mater. Sci. Eng.: C 2004, 24, 39.

19. Kumara, K. K. S.; Geethaa, S.; Trivedi, D. C. Curr. Appl. Phys. 2005, 5, 603.

20. Akagi, K.; Shirakawa, H. Synth. Met. 1993, 60, 85.

21. Cheah, K.; Forsyth, M.; Simon, G. P. Synth. Met. 1999, 102, 1232.

22. Reddy, K. R.; Sin, B. C.; Ryu, K. S.; Noh, J.; Lee, Y. I. Synth. Met. 2009, 159, 1934.

23. Min, Y.; Xia, Y.; MacDiarmid, A. G.; Epstein, A. J. Synth. Met. 1995, 69, 159.

24. Wang, P. C.; MacDiarmid, A. G. React. Funct. Polym. 2008, 68, 201.

25. Ryu, K. S.; Chang, S. H.; Kang, S. G.; Oh, E. J.; Yo, C. H. Bull. Korean Chem. Soc. 1999, 20, 333.

26. Fischer, J. R.; Tang, X.; Scherr, E. M.; Cajipe, V. B.; MacDiarmid, A. G. Synth. Met. 1991, 41, 661.

27. Monkman, A. P.; Adams, P. Synth. Met. 1991, 41, 627.

28. Monkman, A. P.; Adams, P. Synth. Met. 1991, 40, 87.

29. Abell, L.; Pomfret, S. J.; Adams, P. N.; Middleton, A. C.; Monkman, A. P. Synth. Met. 1997, 84, 803. 
30. Kim, W. J.; Kim, T. Y.; Ko, J. W.; Kim, Y. S.; Park, C. M.; Suh, K. S. Trans. KIEE. 2004, 53C, 305.

31. Ruckenstein, E.; Sun, Y. Synth. Met. 1995, 74, 107.

32. Bhadra, S.; Khastgir, D.; Singha, N. K.; Lee, J. H. Prog. Polym. Sci. 2009, 34, 783.

33. MacDiarmid, A. G.; Min, Y.; Wiesinger, J. M.; Oh, E. J.; Scherr, E. M.; Epstein, A. J. Synth. Met. 1993, 55, 753.

34. Oh, E. J.; Min, Y.; Wiesinger, J. M.; Manohar, S. K.; Scherr, E. M.; Prest, P. J.; MacDiarmid, A. G.; Epstein, A. J. Synth. Met. 1993, 55, 977.

35. MacDiarmid, A. G. Synth. Met. 2001, 125, 11.

36. Devendrappa, H.; Subba Rao, U. V.; Ambika Prasad, M. V. N. J. Power Sources 2006, 155, 368.

37. Xia, Y.; Wiesinger, J. M.; MacDiarmid, A. G.; Epstein, A. J.
Chem. Mater. 1995, 7, 443.

38. Tang, J.; Jing, X.; Wang, B.; Wang, F. Synth. Met. 1988, 24, 231.

39. Ruckenstein, E.; Yang, S. Synth. Met. 1993, 53, 283.

40. Koenig, J. L. Infrared and Raman Spectroscopy of Polymers; Rapra, Rev. Rep. 2001, 12(2).

41. Lu, X. F.; Hay, J. N. Polym. 2001, 42, 8055.

42. Nge, T. T.; Hori, N.; Takemura, A.; Ono, H.; Kimura, T. J. Appl. Polym. Sci. 2003, 90, 1932.

43. Hwang, H. Y.; Lee, S. W.; Kim, I. W.; Lee, H. S. Synth. Met. 1995, $69,225$.

44. Shujun, W.; Jinglin, Y.; Wenyuan, G. Am. J. Biochem. Biotechnol. 2005, 1, 207.

45. Wang, Z. H.; Scherr, E. M.; MacDiarmid, A. G.; Epstein, A. J. Phys. Rev. B 1992, 45, 4190. 